\title{
METRNL attenuates lipid-induced inflammation and insulin resistance via AMPK or PPARס-dependent pathways in skeletal muscle of mice
}

Tae Woo Jung ${ }^{1}$, Sung Hoon Lee ${ }^{2}$, Hyoung-Chun Kim³ ${ }^{3}$ Joon Seok Bang ${ }^{4}$, A. M. Abd El-Aty $\mathbb{1}^{5,6}$, Ahmet Hacımüftüoğlu' Yong Kyoo Shin ${ }^{7}$ and Ji Hoon Jeong ${ }^{7}$

\begin{abstract}
Physical activity has many beneficial effects on metabolic disorders, such as obesity, insulin resistance, and diabetes. Meteorin-like protein (METRNL), a novel secreted protein homologous to the neurotrophin Metrn, is induced after exercise in the skeletal muscle. Herein, we investigated the effects of METRNL on lipid-mediated inflammation and insulin resistance in skeletal muscle via AMP-activated protein kinase (AMPK) or peroxisome proliferator-activated receptor $\delta$ (PPARS). Treatment with METRNL suppressed inflammatory markers, such as nuclear factor $\mathrm{KB}$ (NFKB) nuclear translocation, inhibitory $\mathrm{kBa}(\mathrm{I} \mathrm{KBa})$ phosphorylation, interleukin-6 (IL-6) expression, and pro-inflammatory cytokines (such as TNFa and MCP-1). METRNL treatment also attenuated the impaired insulin response both in palmitate-treated differentiated C2C12 cells and the skeletal muscle of high-fat diet (HFD)-fed mice. Furthermore, METRNL administration rescued glucose intolerance and reduced HFD-induced body weight gain in mice; however, METRNL did not affect calorie intake. METRNL treatment increased AMPK phosphorylation and PPARS expression both in differentiated $\mathrm{C} 2 \mathrm{C} 12$ cells and mouse skeletal muscle. siRNA-mediated suppression of AMPK and PPARS abrogated the suppressive effects of METRNL on palmitate-induced inflammation and insulin resistance. Moreover, METRNL augmented the mRNA expression of fatty acid oxidation-associated genes, such as carnitine palmitoyltransferase 1 (CPT1), acyl-CoA oxidase (ACO), and fatty acid binding protein 3 (FABP3). siRNAs for AMPK and PPAR $\delta$ reversed these changes. In the current study, we report for the first time that METRNL alleviates inflammation and insulin resistance and induces fatty acid oxidation through AMPK or PPAR $\delta$-dependent signaling in skeletal muscle.
\end{abstract}

\section{Introduction}

Although regular physical activity appears to have beneficial effects on insulin sensitivity in humans, the potential mechanisms underlying these effects remain to be elucidated ${ }^{1}$. Myokines, such as fibroblast growth factor 21 (FGF21), irisin, $\beta$-aminoisobutyric acid (BAIBA), and

\footnotetext{
Correspondence: Yong Kyoo Shin (syk@cau.ac.kr) or Ji Hoon Jeong

(jhjeong3@cau.ac.kr)

${ }^{1}$ Research Administration Team, Seoul National University Bundang Hospital, Seongnam, Korea

${ }^{2}$ College of Pharmacy, Chung-Ang University, Seoul, Korea

Full list of author information is available at the end of the article.
}

meteorin-like protein (METRNL), may partly mediate the beneficial effects of regular exercise on whole-body function ${ }^{2}$. METRNL, also known as subfatin, is a circulating protein expressed in monocytes, adipocytes, and skeletal muscle ${ }^{3,4}$. METRNL is induced in skeletal muscle upon exercise ${ }^{5}$ and in white adipose tissue upon cold exposure ${ }^{6}$. METRNL elevates whole-body energy expenditure and improves glucose tolerance by enhancing the browning of white adipose tissue via a STAT6-mediated pathway in adipose tissue infiltrated by macrophages ${ }^{6}$. Furthermore, METRNL transgenic mice have shown that METRNL improves insulin sensitivity through activation 
of PPAR $\gamma$-mediated signaling, which was assumed to play a crucial role in the regulation of adipocyte differentiation $^{5,7}$. However, the direct effects of METRNL on inflammation and insulin signaling in skeletal muscle and its underlying mechanisms remain unknown.

Increased serum-free fatty acid levels are detected in patients with insulin resistance ${ }^{8}$. Saturated free fatty acid causes impaired insulin signaling ${ }^{9}$ and inflammation, leading to insulin resistance through various pathways associated with diacylglycerol-mediated protein kinase $\mathrm{C}^{10}$, Toll-like receptor (TLR)- $2^{11}$, or TLR- $4^{12}$. These pathways activate nuclear factor $\kappa B(\mathrm{NF} \kappa \mathrm{B})$, a well-known pro-inflammatory transcription factor that induces insulin resistance in skeletal muscle ${ }^{13}$. Activation of the NFkBmediated pathway stimulates the expression of proinflammatory cytokines, such as TNF $\alpha$ and IL-6, which play a crucial role in the development of insulin resistance and type 2 diabetes $^{14}$. Therefore, proper suppression of lipid-induced inflammation is of critical importance for the treatment of diabetes.

In the present study, we investigated the effects of the novel myokine, METRNL, on lipid-induced inflammation and insulin resistance. Additionally, we explored a downstream pathway related to AMP-activated protein kinase (AMPK) and peroxisome proliferator-activated receptor $\delta$ (PPAR $\delta$ ) in differentiated $\mathrm{C} 2 \mathrm{C} 12$ cells and mouse skeletal muscle.

\section{Materials and methods}

\section{Cell cultures, reagents, and antibodies}

The mouse skeletal muscle cell line C2C12 (ATCC, Manassas, VA, USA) was cultured in Dulbecco's modified eagle medium (DMEM; Invitrogen, Carlsbad, CA, USA) supplemented with $10 \%$ fetal bovine serum (Invitrogen), 100 units $/ \mathrm{mL}$ penicillin, and $100 \mu \mathrm{g} / \mathrm{mL}$ streptomycin (Invitrogen). Cells were cultured and maintained at $37^{\circ} \mathrm{C}$ in a humidified atmosphere of $5 \%$ $\mathrm{CO}_{2}$. Cells were supplemented with $2 \%$ horse serum (for $48 \mathrm{~h})$ to induce differentiation. $\mathrm{C} 2 \mathrm{C} 12$ cells were confirmed to be free from mycoplasma. We used cells at passages 5-10 for all experiments. Mouse recombinant METRNL (Adipogen, San Diego, CA, USA) was dissolved in phosphate-buffered saline (PBS). Sodium palmitate (Sigma, St Louis, MO, USA) was conjugated to $2 \%$ BSA (fatty acid free grade; Sigma) dissolved in DMEM. In all experiments, cells were treated with palmitate-BSA for $24 \mathrm{~h}$, and $2 \%$ BSA was used as a control. Cells were treated with $0-200 \mathrm{ng} / \mathrm{mL} \mathrm{METRNL}^{7}$ and $200 \mu \mathrm{M}$ palmitate for $24 \mathrm{~h}$ without palmitate pretreatment or additional treatment steps. Cells were cultured in serum starvation media (without FBS) for $6 \mathrm{~h}$ before insulin treatment. Insulin $(10 \mathrm{nM})$ was used to stimulate insulin signaling, IRS-1 and Akt for 3 min after METRNL and palmitate treatment.

\section{Animals, feeding, and treatment}

This study was approved by the Institutional Animal Review Board of Chung Ang University, Seoul, Republic of Korea. Animal studies were conducted in accordance with the Guide for the Care and Use of Laboratory Animals (NIH publication, 8th edition, 2011).

\section{Experiment 1}

A control and two experimental groups of 8-week-old male C57BL/6J (B6) mice were given a normal diet (ND; Brogaarden, Gentofte, Denmark) or a high-fat diet (HFD; Research Diets, New Brunswick, NJ, USA) for 8 weeks. The HFD plus METRNL group was additionally administered METRNL intravenously $\left(2 \mu \mathrm{g} / \mathrm{mouse}_{\mathrm{day}}\right)^{7}$, and the ND and HFD groups were administered vehicle intravenously at the same volume (mouse/day) for 8 weeks. Mouse soleus skeletal muscle samples were isolated $10 \mathrm{~min}$ after intraperitoneal injection of insulin (Novo Nordisk, Princeton, NJ, USA; $10 \mathrm{U} / \mathrm{kg}$ body weight). The intraperitoneal glucose tolerance test (IPGTT) was performed as follows: mice were fasted for $12 \mathrm{~h}$ (overnight) and then intraperitoneally injected with glucose ( $2 \mathrm{~g} / \mathrm{kg}$ body weight). Thereafter, serum glucose levels were measured at $0,30,60,90$, and $120 \mathrm{~min}$ post administration. To perform the insulin tolerance test (ITT), mice were fasted for $6 \mathrm{~h}$ and then given an intraperitoneal injection of human insulin $(1 \mathrm{U} / \mathrm{kg}$ body weight). Serum glucose levels were measured at $0,15,30$, 45 , and $60 \mathrm{~min}$ thereafter. The IPGTT was performed 3 days before the mice treated with HFD and METRNL for 8 weeks were sacrificed. One day after the end of the IPGTT, the ITT was performed. Serum glucose levels were measured using an Accu-Check III glucose analyzer.

\section{Experiment 2}

A control and two experimental groups of 8-week-old male C57BL/6J (B6) mice were given a normal diet (ND; Brogaarden, Gentofte, Denmark) or a high-fat diet (HFD; Research Diets, New Brunswick, NJ, USA) for 2 weeks. The HFD plus METRNL group was additionally administered METRNL intravenously ( $2 \mu \mathrm{g} /$ mouse/day), and the ND and HFD groups were administered vehicle intravenously at the same volume (mouse/day) for 2 weeks. AMPK siRNA $(100 \mu \mathrm{g} /$ mouse $)$ or PPAR $\delta$ siRNA ( $80 \mu \mathrm{g} /$ mouse) was injected twice (at 48 -h intervals) for 2 weeks into the HFD-fed and METRNL-treated AMPK or PPAR $\delta$-knockdown groups through the tail vein using in vivo-jetPEI ${ }^{\mathrm{TM}}$ (Polyplus Transfection, NY, USA) for siRNA delivery into skeletal muscle. Scrambled siRNA was injected twice into each mouse in the ND group. Upon completion of the study period, all experimental mice were sacrificed under anesthesia after fasting overnight for $12 \mathrm{~h}$. 


\section{RNA extraction and quantitative real-time PCR}

Total RNA from harvested $\mathrm{C} 2 \mathrm{C} 12$ cells and soleus skeletal muscle tissue was isolated using TRIzol reagent (Invitrogen, Carlsbad, CA). Gene expression was measured by quantitative real-time PCR (qPCR) using the fluorescent TaqMan 5' nuclease assay on an Applied Biosystems 7000 sequence detection system (Foster City, CA, USA). qPCR was performed using cDNA with $2 \times$ TaqMan Master Mix and the $20 \times$ premade TaqMan gene expression assays (Applied Biosystems). qPCR conditions were $95^{\circ} \mathrm{C}$ for $10 \mathrm{~min}$, followed by 40 cycles of $95^{\circ} \mathrm{C}$ for $15 \mathrm{~s}$ and $60^{\circ} \mathrm{C}$ for $1 \mathrm{~min}$. Expression of mouse carnitine palmitoyltransferase 1 (CPT1) (Mm00463960_ml; Applied Biosystems), acyl-CoA oxidase (ACO) (Mm00801417_ml), fatty acid binding protein 3 (FABP3) (Mm02342495_ml), and spliced XBP-1 mRNA was normalized to the expression of mouse beta-actin (Mm00607939_sl; Applied Biosystems). Mouse spliced XBP-1 primers (forward: 5'GAGTCCGCAGCAGGTG-3' and reverse: 5'-GTGT CAGAGTCCATGGGA-3') were used.

\section{Western blot analysis}

Differentiated $\mathrm{C} 2 \mathrm{C} 12$ cells were harvested, and proteins were extracted with lysis buffer (PRO-PREP; Intron Biotechnology, Seoul, Republic of Korea) for $60 \mathrm{~min}$ at $4{ }^{\circ} \mathrm{C}$. Protein samples $(30 \mu \mathrm{g})$ were subjected to $12 \%$ SDSPAGE, transferred to a nitrocellulose membrane (Amersham Bioscience, Westborough, MA, USA), and probed with the indicated primary antibody followed by a secondary antibody conjugated with horseradish peroxidase (Santa Cruz Biotechnology). The signals were detected using enhanced chemoluminescence (ECL) kits (Amersham Bioscience). Anti-insulin receptor substrate (IRS)-1 (1:2500), anti-phospho-Akt (Ser473; 1:1000), anti-Akt (1:1000), anti-phospho-AMPK (Thr172; 1:1000), antiAMPK (1:2500), anti-NFkBp65 (1:2500), anti-phosphoIкB $\alpha$ (Ser32; 1:1000), anti-phospho-eIF2 $\alpha$ (Ser51; 1:1000), anti-eIF2 $\alpha$ (1:1000), anti-PPAR $\delta$ (1:2500), anti-PPAR $\alpha$ (1:1000), anti-PPARY (1:1000), anti-phospho-LKB1 (Ser428; 1:1000), anti-LKB1 (1:2500), anti-phosphoacetyl-CoA carboxylase (ACC) (Ser79; 1:1000), antiACC (1:2500), and anti-PGC1 $\alpha$ (1:1000) antibodies were procured from Cell Signaling Technology (Beverly, MA, USA). Anti-phospho-IRS-1 (Tyr632; 1:1000) and antibeta-actin (1:5000) antibodies were obtained from Santa Cruz Biotechnology (Santa Cruz, CA, USA). The antiMETRNL antibody (1:1000) was supplied by Abcam (Cambridge, MA, USA).

\section{Enzyme-linked immunosorbent assay (ELISA)}

The concentrations of METRNL, TNF $\alpha$, and MCP-1 in mouse serum were each measured using an ELISA kit (R\&D Systems, Minneapolis, MN, USA) according to the manufacturer's instructions.

\section{Transfection with siRNAs for gene silencing}

Small interfering (si) RNA oligonucleotides $(20 \mathrm{nM})$ specific for AMPK and PPAR $\delta$ were acquired from Santa Cruz Biotechnology. To suppress gene expression, cell transfection was performed with Lipofectamine 2000 (Invitrogen) in accordance with the manufacturer's instructions. In brief, the cells were grown to 50-60\% confluence and fully differentiated, followed by serum starvation for $6 \mathrm{~h}$. The cells were transfected with validated siRNA or scramble siRNA at a final concentration of 20 $\mathrm{nM}$ in the presence of the transfection reagent. After transfection, the cells were harvested at $36 \mathrm{~h}$ for protein extraction or further treated with $10 \mathrm{nM}$ insulin, $20 \mu \mathrm{g} / \mathrm{mL}$ METRNL, or $200 \mu \mathrm{M}$ palmitate.

\section{Cell fractionation for the measurement of NFKB nuclear translocation}

Cells were fractionated using a Nuclear/Cytosol Fractionation Kit according to the manufacturer's protocols (Bioviosion, Milpitas, CA, USA).

\section{Measurement of glucose uptake, acetyl-CoA, and ATP content}

Glucose uptake levels were measured using the Glucose Uptake Assay $\mathrm{Kit}^{\mathrm{TM}}$ (Abcam). In brief, proliferating C2C12 cells were seeded at $5 \times 10^{4}$ cells/well in blackwalled/clear-bottom 96-well plates (Corning, Inc., Corning, NY, USA) in DMEM containing 10\% FBS. Upon reaching a confluency of $50 \%$, differentiation was induced with differentiating media consisting of high-glucose DMEM and 2\% horse serum. After $48 \mathrm{~h}$, the media was changed to media containing palmitate $(250 \mu \mathrm{M})$ or METRNL (0-200 ng/mL) for $24 \mathrm{~h}$. Following treatment, the media was removed from the wells, and the cells were treated with $10 \mathrm{nM}$ insulin and $1 \mathrm{mM}$ 2-deoxyglucose (2DG) for $30 \mathrm{~min}$. Afterward, the plates were centrifuged for $1 \mathrm{~min}$ at $500 \mathrm{rpm}$ and incubated for $1 \mathrm{~h}$ at room temperature. After 2-DG was taken up by the cells, it was extracted by extraction buffer in the kit. 2-DG uptake levels were measured at a wavelength of OD $412 \mathrm{~nm}$ on a BioTek Synergy HT plate reader (BioTek Instruments, Inc., Winooski, VT, USA). Intracellular levels of acetylCoA were measured using the PicoProbe Acetyl CoA Assay $\mathrm{Kit}^{\mathrm{TM}}$ (Abcam), and intracellular ATP levels were measured using ATP Assay $\mathrm{Kit}^{\mathrm{TM}}$ (Abcam) in differentiated $\mathrm{C} 2 \mathrm{C} 12$ cells or soleus skeletal muscle according to the manufacturer's instructions.

\section{Statistical analysis}

All statistical analyses were conducted using the SPSS/ PC statistical program (version 12.0 for Windows; SPSS, Chicago, IL, USA). The results are presented as the fold change of the highest value (mean \pm SEM). All of the in vitro experiments were performed at least three times. 


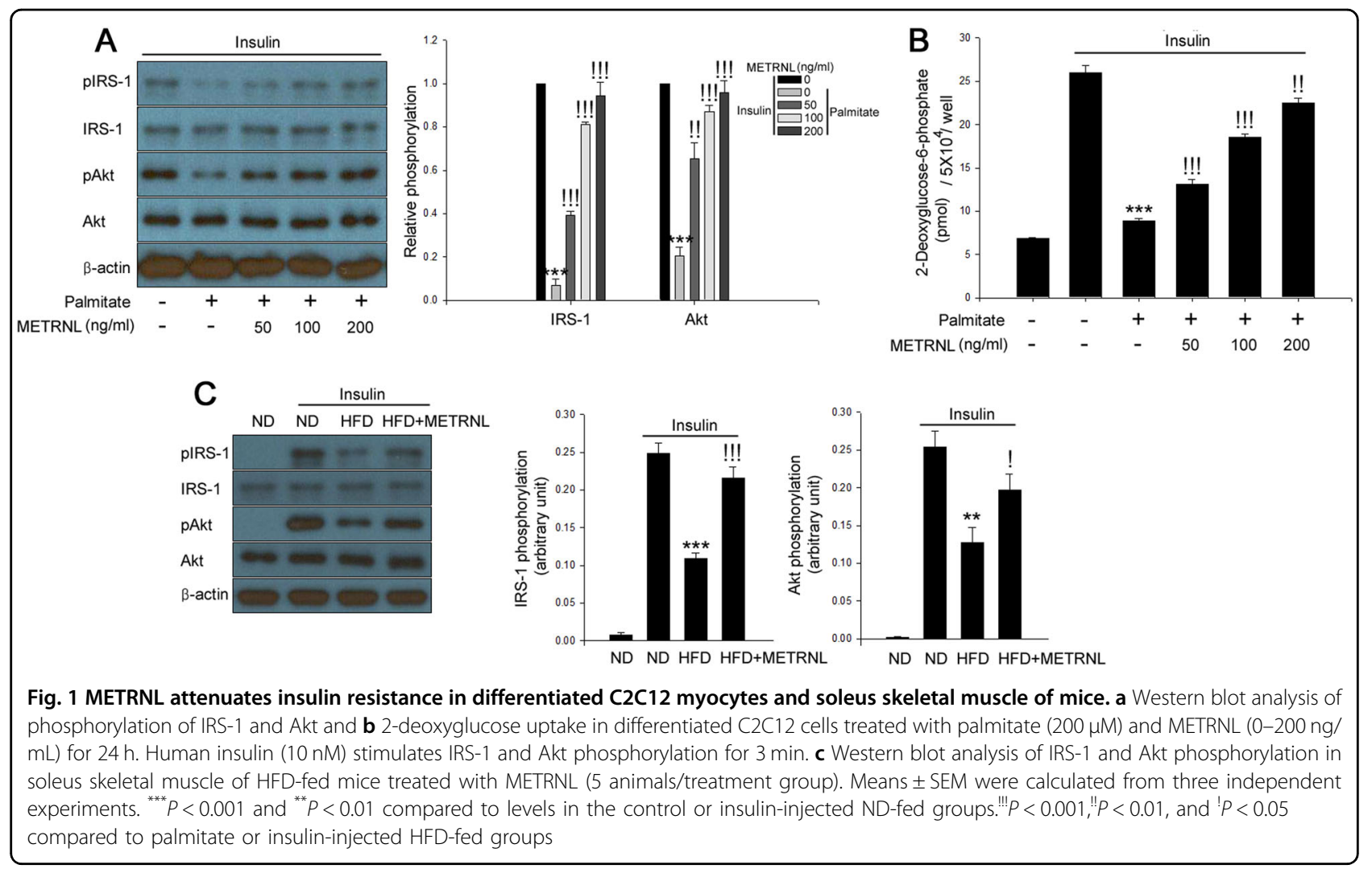

All of the in vivo experiments were performed with five (experiment 1) or three (experiment 2) mice in each group. One-way ANOVA with Tukey's post hoc test was used for statistical analysis.

\section{Results}

METRNL prevents impairment of insulin sensitivity in palmitate-treated $\mathrm{C} 2 \mathrm{C} 12$ cells and skeletal muscle of HFDfed mice

We first evaluated METRNL expression in palmitatetreated $\mathrm{C} 2 \mathrm{C} 12$ cells and soleus skeletal muscle of HFD-fed mice to reveal its relevance under hyperlipidemic conditions. In agreement with a recent report ${ }^{15}$, HFD treatment decreased METRNL expression in skeletal muscle and its circulating levels in mice. Furthermore, treatment of $\mathrm{C} 2 \mathrm{C} 12$ myocytes with palmitate significantly suppressed METRNL expression (Suppl. Fig. 1). Afterward, we selected and optimized the appropriate concentrations of METRNL $^{7}$. To investigate the effects of METRNL on palmitate-induced insulin resistance, we examined the effect of METRNL on insulin-stimulated IRS-1 and Akt phosphorylation and glucose uptake. Differentiated $\mathrm{C} 2 \mathrm{C} 12$ cells treated with palmitate and soleus skeletal muscle of HFD-fed mice exhibited an impaired insulin response. However, METRNL treatment markedly restored these changes in both $\mathrm{C} 2 \mathrm{C} 12$ cells (Fig. 1a, b) and isolated soleus skeletal muscle of HFD-fed mice (Fig. 1c).
METRNL attenuates HFD-induced insulin resistance in mice

We next investigated the effects of METRNL on glucose tolerance and insulin sensitivity by performing the IPGTT and ITT. These tests revealed that HFD markedly aggravated glucose tolerance and decreased insulin sensitivity compared with ND. However, METRNL administration ameliorated HFD-induced glucose tolerance and insulin resistance (Fig. 2a, b). As the HFD-induced basal serum glucose levels were decreased by METRNL treatment; the ITT results were difficult to interpret. Therefore, we normalized the results to basal glycemia to investigate the effects of METRNL on insulin sensitivity (Fig. 2b). Moreover, METRNL treatment decreased HFD-induced body weight gain (Fig. 2c) but had no effect on calorie intake (Fig. 2d).

\section{METRNL ameliorates palmitate-induced inflammation in C2C12 myocytes and soleus skeletal muscle of HFD-fed mice}

METRNL attenuated palmitate-induced $N_{K} B$ nuclear translocation, IкB $\alpha$ phosphorylation, and IL- 6 expression in a dose-dependent manner (Fig. 3a). Furthermore, treatment with METRNL attenuated the HFD-induced $\mathrm{NF} \kappa \mathrm{B}$ nuclear translocation and I $\mathrm{B} \alpha$ phosphorylation in mouse skeletal muscle (Fig. 3b). Serum TNF $\alpha$ and monocyte chemotactic factor-1 (MCP-1) were also reduced by METRNL administration (Fig. 3c, d). 

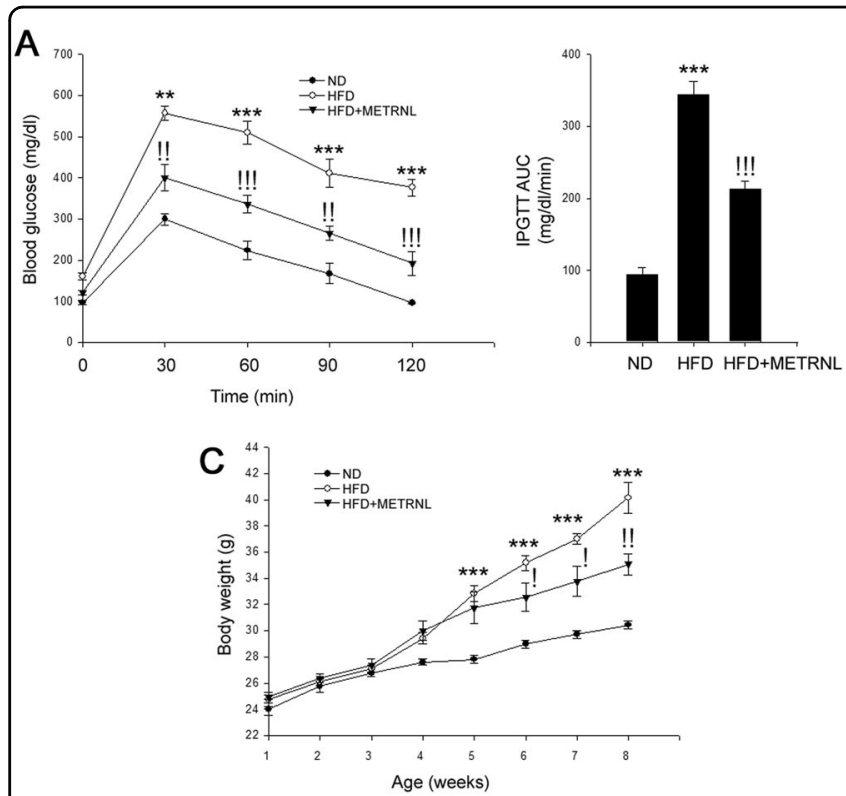
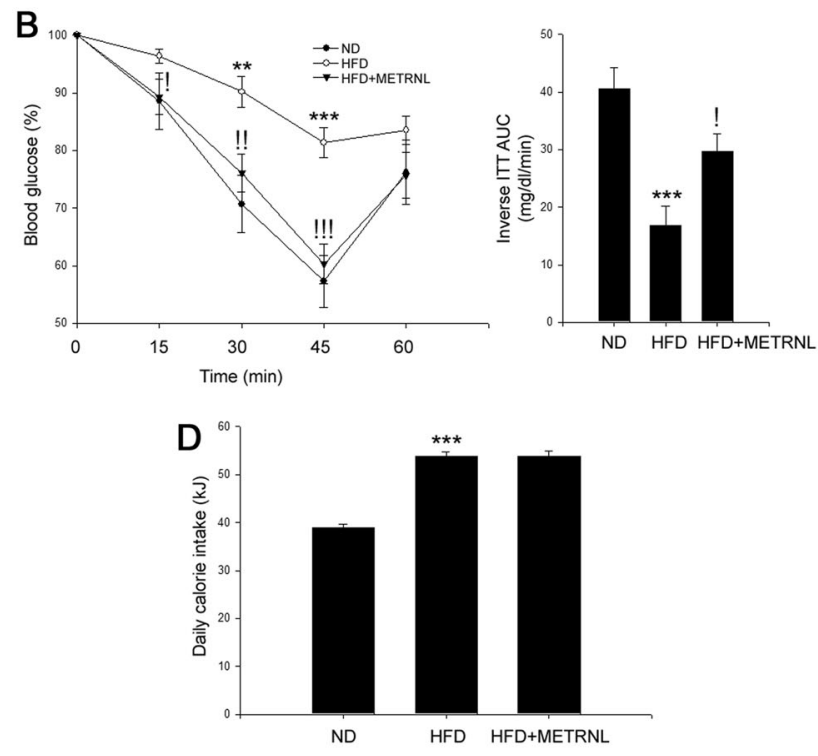

Fig. 2 METRNL administration attenuates insulin resistance in mice. a The IPGTT of experimental mice and the glucose area under the curve (AUC) during the IPGTT. $\mathbf{b}$ The ITT and the glucose inverse AUC during the ITT. c Body weight measurement and $\mathbf{d}$ daily energy intake in mice (5 animals/treatment group). Closed circles, ND; opened circles, HFD; closed triangles, HFD + METRNL. Means \pm SEM were calculated from five separate animals. ${ }^{* * *} P<0.001$ and ${ }^{* *} P<0.01$ compared to ND treatment.!" $P<0.001,{ }^{\prime \prime} P<0.01$ and $! P<0.05$ compared to HFD treatment

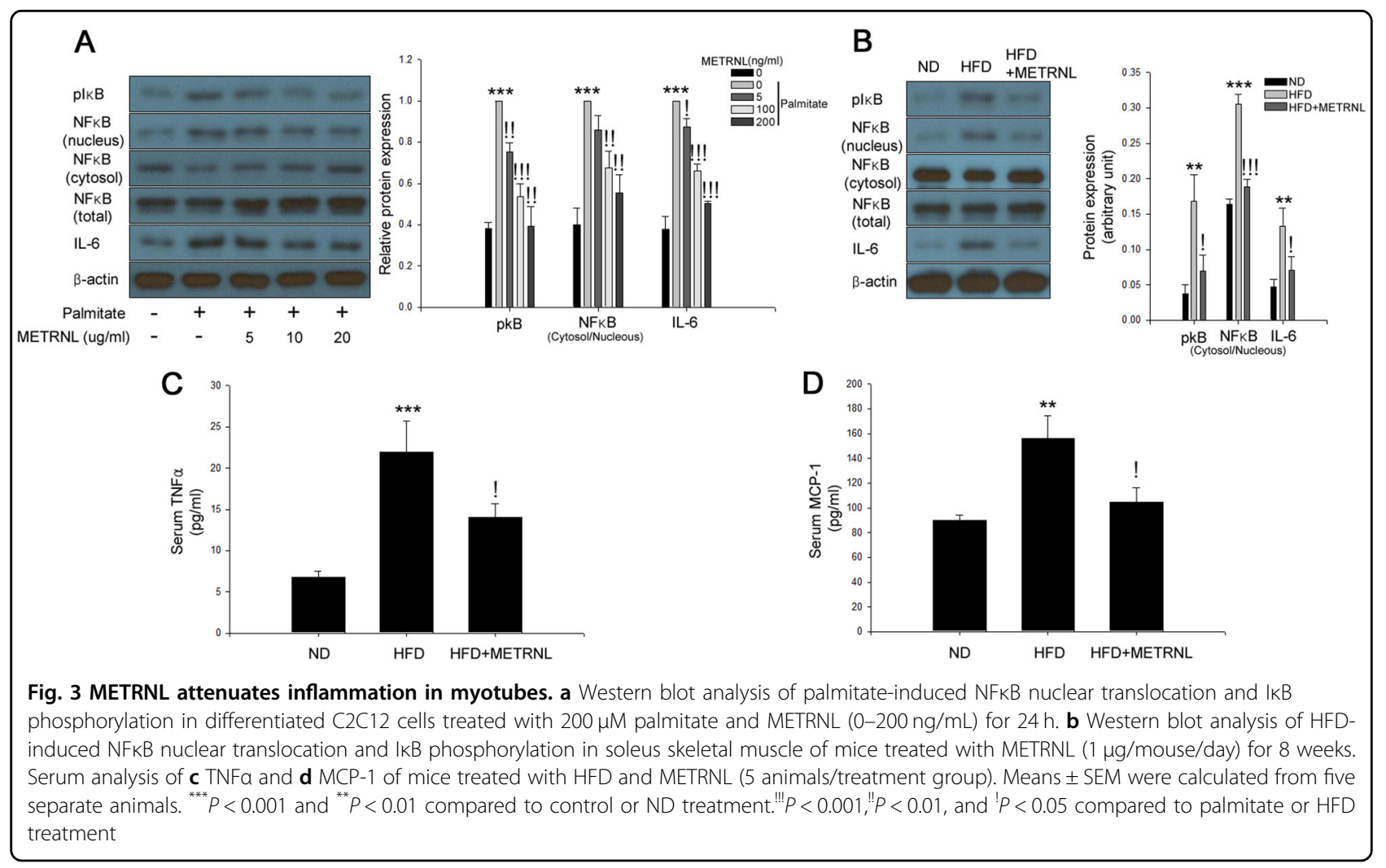



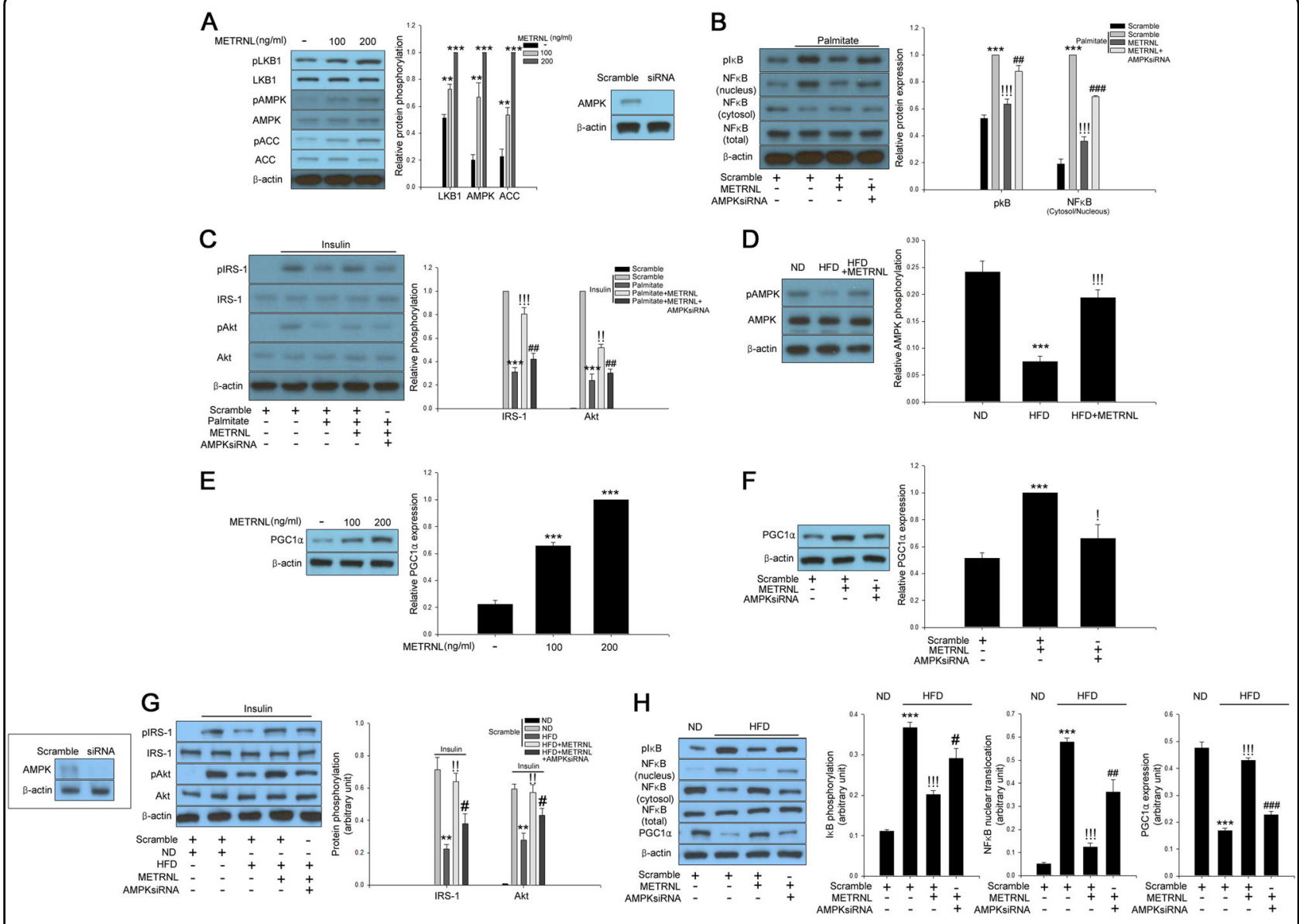

Fig. 4 METRNL ameliorates inflammation and insulin resistance via an AMPK-mediated pathway. a Western blot analysis of LKB1, AMPK, and ACC phosphorylation in differentiated C2C12 cells treated with METRNL $(0-200 \mathrm{ng} / \mathrm{mL})$ for $24 \mathrm{~h}$. b Confirmation of AMPK siRNA efficiency in differentiated C2C12 cells. Western blot analysis of palmitate (200 $\mu$ M)-induced inflammation markers in AMPK siRNA (20 nM)-transfected differentiated C2C12 cells treated with METRNL (0-200 ng/mL) for $24 \mathrm{~h}$. c Western blot analysis of the palmitate-induced impairment of IRS-1 and Akt phosphorylation in AMPK siRNA-transfected differentiated C2C12 cells treated with METRNL (0-200 ng/mL) for $24 \mathrm{~h}$. Human insulin (10 nM) was used to stimulate insulin signaling for $3 \mathrm{~min}$. $\mathbf{d}$ Western blot analysis of AMPK phosphorylation in soleus muscle of mice treated with HFD and METRNL ( 5 animals/treatment group). e Western blot analysis of PGC1a expression in differentiated C2C12 cells treated with METRNL (0-200 ng/mL) for $24 \mathrm{~h}$. f Western blot analysis of METRNL ( $200 \mathrm{ng} / \mathrm{mL}$ )-induced PGC1a expression in AMPK siRNA (20 nM)-transfected differentiated C2C12 cells for $24 \mathrm{~h}$. Confirmation of AMPK siRNA efficiency in skeletal muscle of mice. Western blot analysis of IRS-1 and Akt phosphorylation (g), and inflammatory markers (h) in AMPK siRNA-transfected skeletal muscle of experimental mice. Means \pm SEM were obtained from three separate experiments or five animals. ${ }^{* * *} P<0.001,{ }^{* *} P<0.01$, and ${ }^{*} P<0.05$ compared to control or ND treatment."' $P<0.001$ and $"$ " $P<0.01$ compared to palmitate or HFD treatment. $\# \#<0.001, \# P<0.01$, and ${ }^{\#} P<0.05$ compared to palmitate plus METRNL treatment or HFD plus KA group

\section{METRNL treatment attenuates hyperlipidemia-induced} inflammation, which results in the amelioration of insulin

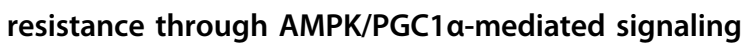

AMPK has been reported to prevent inflammation ${ }^{16}$. Furthermore, AMPK has been suggested as a therapeutic target for treating insulin resistance and type 2 diabetes ${ }^{17}$. In the present study, the treatment of differentiated C2C12 cells with recombinant METRNL induced phosphorylation of AMPK, as well as ACC, a downstream substrate of AMPK, and LKB1, an upstream AMPK kinase, in a dose-dependent manner (Fig. 4a). We next confirmed the suppressive effects of METRNL on palmitate-induced inflammation through the AMPK- mediated pathway. As shown in Fig. 4b, siRNAmediated suppression of AMPK mitigated the effects of METRNL on palmitate-induced NFKB nuclear translocation and $\mathrm{I} \kappa \mathrm{B} \alpha$ phosphorylation (Fig. 4b). We then examined whether METRNL-induced AMPK contributed to the attenuation of insulin resistance caused by palmitate in $\mathrm{C} 2 \mathrm{C} 12$ cells. As shown in Fig. 4c, treatment of C2C12 cells with METRNL markedly suppressed palmitate-induced insulin resistance, as detected by an impairment of insulin-stimulated IRS-1 and Akt phosphorylation. In contrast, AMPK siRNA markedly mitigated the effects of METRNL on palmitate-induced insulin resistance (Fig. 4c). METRNL administration 
markedly reversed the HFD-induced suppression of AMPK phosphorylation in mouse skeletal muscle (Fig. 4d). Exercise-induced PGC1 $\alpha$ plays a crucial role in the regulation of muscle metabolism and stimulates the release of METRNL from skeletal muscle ${ }^{6}$. AMPK stimulates PGC1 $\alpha$-mediated signaling ${ }^{18,19}$. PGC1 $\alpha$ has previously been shown to exhibit powerful suppressive effects on inflammation ${ }^{20}$ and insulin resistance ${ }^{21}$. Thus, we further examined the effects of METRNL on PGC1 $\alpha$ expression. Treatment of $\mathrm{C} 2 \mathrm{C} 12$ myocytes with METRNL increased PGC1 $\alpha$ expression in a dose-dependent fashion (Fig. 4e). Furthermore, AMPK siRNA abrogated the effects of METRNL on PGC1 $\alpha$ expression (Fig. 4f), proving that METRNL induces PGC1 $\alpha$ expression through AMPK signaling. To establish the possible links between METRNL, inflammation and insulin resistance more clearly, we performed preliminary animal experiments. To reproduce METRNL-mediated cellular signaling in vitro, experimental mice were treated with HFD and METRNL for 2 weeks. Similar to the in vitro results, suppression of AMPK by in vivo transfection abrogated the effects of METRNL on insulin signaling, inflammation, and PGC1 $\alpha$ expression in skeletal muscle of HFDfed mice (Fig. 4g, h).

\section{PPARס/PGC1a signaling plays an important role in the suppressive effects of METRNL on palmitate-induced inflammation and insulin resistance}

Activation of AMPK has been documented to induce fatty acid oxidation through PPAR $\alpha$ and the $\delta$-dependent pathway $^{22,23}$. Furthermore, PPAR $\alpha, \gamma$, and $\delta$-mediated signaling has been suggested as a target for treating inflammation ${ }^{24-26}$ and insulin resistance ${ }^{27,28}$. Therefore, we investigated whether METRNL can increase PPAR expression in differentiated $\mathrm{C} 2 \mathrm{C} 12$ cells. Treatment of C2C12 myocytes with METRNL did not show any significant effects on PPAR $\alpha$ or PPAR $\gamma$ expression (Suppl. Figure 2). However, treatment of $\mathrm{C} 2 \mathrm{C} 12$ cells with METRNL augmented PPAR $\delta$ expression in a dosedependent manner (Fig. 5a). siRNA targeting PPAR $\delta$ markedly blocked the effects of METRNL on palmitateinduced inflammation and insulin resistance (Fig. 5b, c), demonstrating that METRNL ameliorates palmitateinduced inflammation and insulin resistance through PPAR $\delta$-dependent signaling. In agreement with the data from in vitro experiments, METRNL treatment markedly increased the expression of PPAR $\delta$ in skeletal muscle of HFD-fed mice (Fig. 5d). PPAR $\delta$ has been reported to activate PGC1 $\alpha$-dependent signaling ${ }^{29}$. siRNA-mediated suppression of PPAR $\delta$ inhibited METRNL-induced PGC1 $\alpha$ expression (Fig. 5e), indicating that PPAR $\delta$ could contribute to METRNL-induced PGC1 $\alpha$ expression. siRNA for PPAR $\delta$ did not affect METRNL-induced AMPK phosphorylation. Moreover, AMPK siRNA also did not influence METRNL-induced PPAR $\delta$ expression in C2C12 cells (Fig. 5f, g). Similar to the in vitro results, suppression of PPAR $\delta$ by in vivo transfection mitigated the effects of METRNL on insulin signaling, inflammation, and PGC1 $\alpha$ expression (Fig. 5h, i). Similar to the results seen in (Fig. 5f, g) knockdown of PPAR $\delta$ did not affect METRNL-induced AMPK phosphorylation (Fig. 5j). Furthermore, AMPK suppression did not influence METRNL-induced PPAR $\delta$ expression in skeletal muscle of HFD-fed mice (Fig. 5k).

METRNL stimulates fatty acid oxidation via an AMPK- or PPARס-dependent pathway in differentiated $\mathrm{C} 2 \mathrm{C} 12$ cells and in soleus skeletal muscle of HFD-fed mice

Koves et al. demonstrated that impaired fatty acid oxidation caused insulin resistance in skeletal muscle ${ }^{30}$. AMPK and PPAR $\delta$ have been documented to induce fatty acid oxidation $^{22,31}$. Therefore, we evaluated whether METRNL-induced AMPK and PPAR $\delta$ could enhance fatty acid oxidation in differentiated $\mathrm{C} 2 \mathrm{C} 12$ cells. METRNL treatment induced the mRNA expression of fatty acid oxidation-associated genes, such as CPT1, ACO, and FABP3, in $\mathrm{C} 2 \mathrm{C} 12$ cells. Suppression of AMPK and PPAR $\delta$ expression by siRNAs significantly abrogated the inducible effects of METRNL on fatty acid oxidation-associated mRNA expression (Fig. 6a). Moreover, METRNL administration increased CPT1, ACO, and FABP3 mRNA expression in skeletal muscle of mice (Fig. 6b). Fatty acid oxidation products (acetyl-CoA and ATP) were further determined. METRNL markedly increased the levels of intracellular acetyl-CoA and ATP (Fig. 6c, d).

\section{METRNL did not affect palmitate-induced endoplasmic reticulum (ER) stress in differentiated $\mathrm{C} 2 \mathrm{C} 12$ cells}

ER stress ${ }^{32}$ has been stated to be is a causative factor in the development of insulin resistance by fatty acids in skeletal muscle. Thus, we next examined whether METRNL suppressed palmitate-induced ER stress. Treatment of differentiated $\mathrm{C} 2 \mathrm{C} 12$ myocytes with palmitate markedly increased the expression of spliced XBP1, an ER stress marker. However, METRNL treatment did not attenuate these palmitate-induced changes (Fig. 7).

\section{Discussion}

Regular physical activity has been demonstrated to have a beneficial effect on insulin sensitivity ${ }^{1}$. During exercise, mRNA expression and release of METRNL from skeletal muscle into the blood are stimulated via PGC1 $\alpha$-mediated signaling activation ${ }^{6}$, which is known to be a key regulator of muscle hypertrophy ${ }^{33}$. METRNL plays an important role in browning white adipose tissue and insulin sensitization via regulation of macrophage activity ${ }^{5}$. Therefore, Rao et al. suggested that METRNL may offer exercisemediated protection against metabolic disorders ${ }^{6}$. 


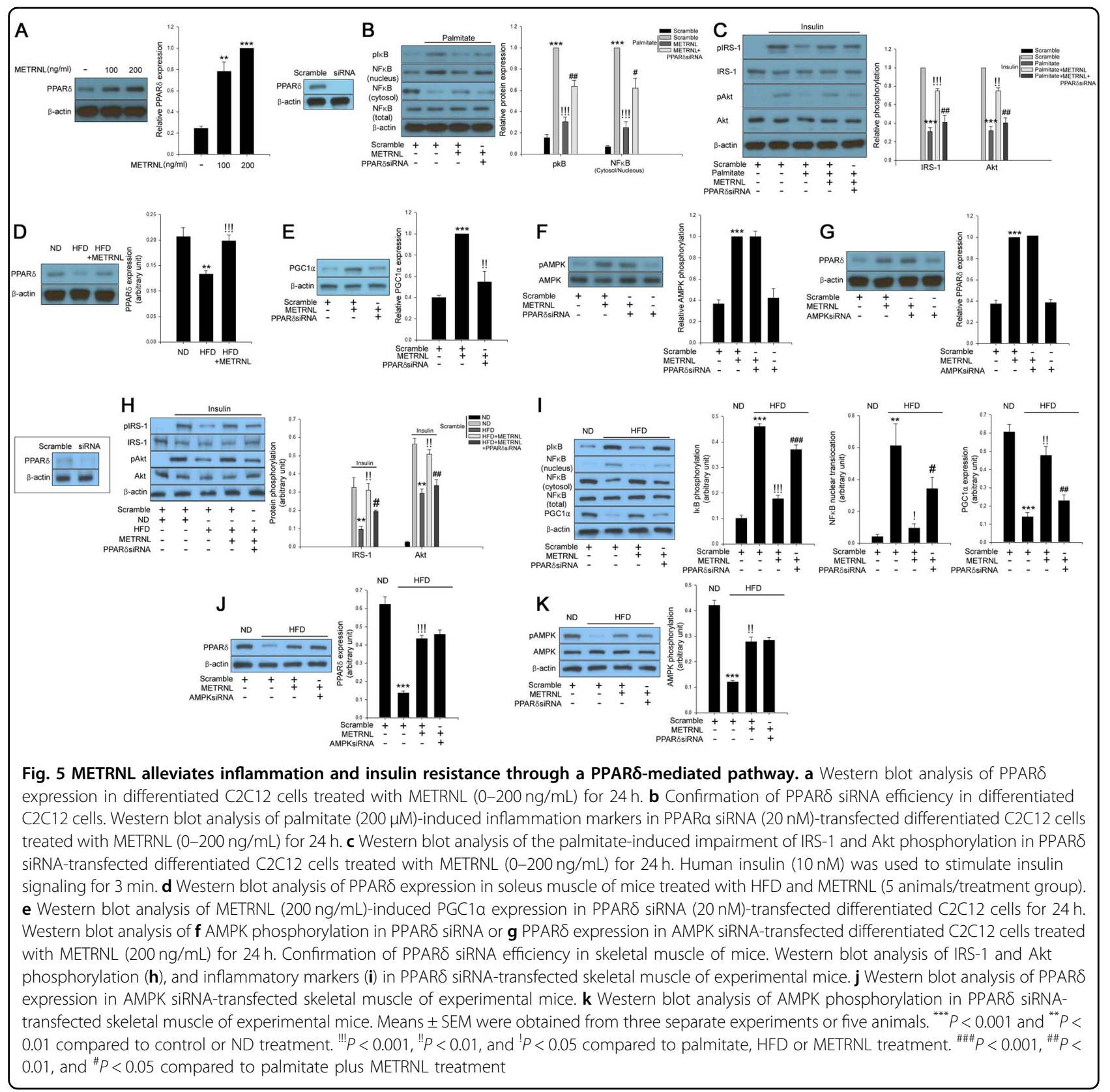

However, the underlying mechanisms by which METRNL may attenuate inflammation and insulin resistance in skeletal muscle remain uncertain.

AMPK plays a pivotal role in cellular energy homeostasis. Additionally, AMPK exerts a significant antiinflammatory effect via suppression of the NFkB signaling pathway $^{16}$ and immunosuppressive effects ${ }^{34}$. Dysregulation of AMPK plays a critical role in the pathogenesis of insulin resistance and metabolic syndrome-associated diseases in humans as well as in experimental models ${ }^{35}$. Elevated serum free fatty acid levels induced by obesity reduce AMPK activity ${ }^{36}$ and insulin sensitivity ${ }^{37,38}$.
Hyperglycemia causes pro-inflammatory actions and oxidative stress ${ }^{39}$. However, activation of AMPK suppresses the production of reactive oxygen species ${ }^{40}$ and mitigates the inflammatory response through induction of thioredoxin. AMPK activation by PPAR $\delta$ also mitigates ER stress, resulting in attenuation of inflammation and insulin resistance ${ }^{41}$. Increases in AMPK activity were detected in the muscle of patients with type 2 diabetes during physical activity ${ }^{42}$. In the present study, we demonstrated that METRNL markedly augmented AMPK phosphorylation. Additionally, AMPK siRNA blocked the inhibitory effects of METRNL on inflammation and 

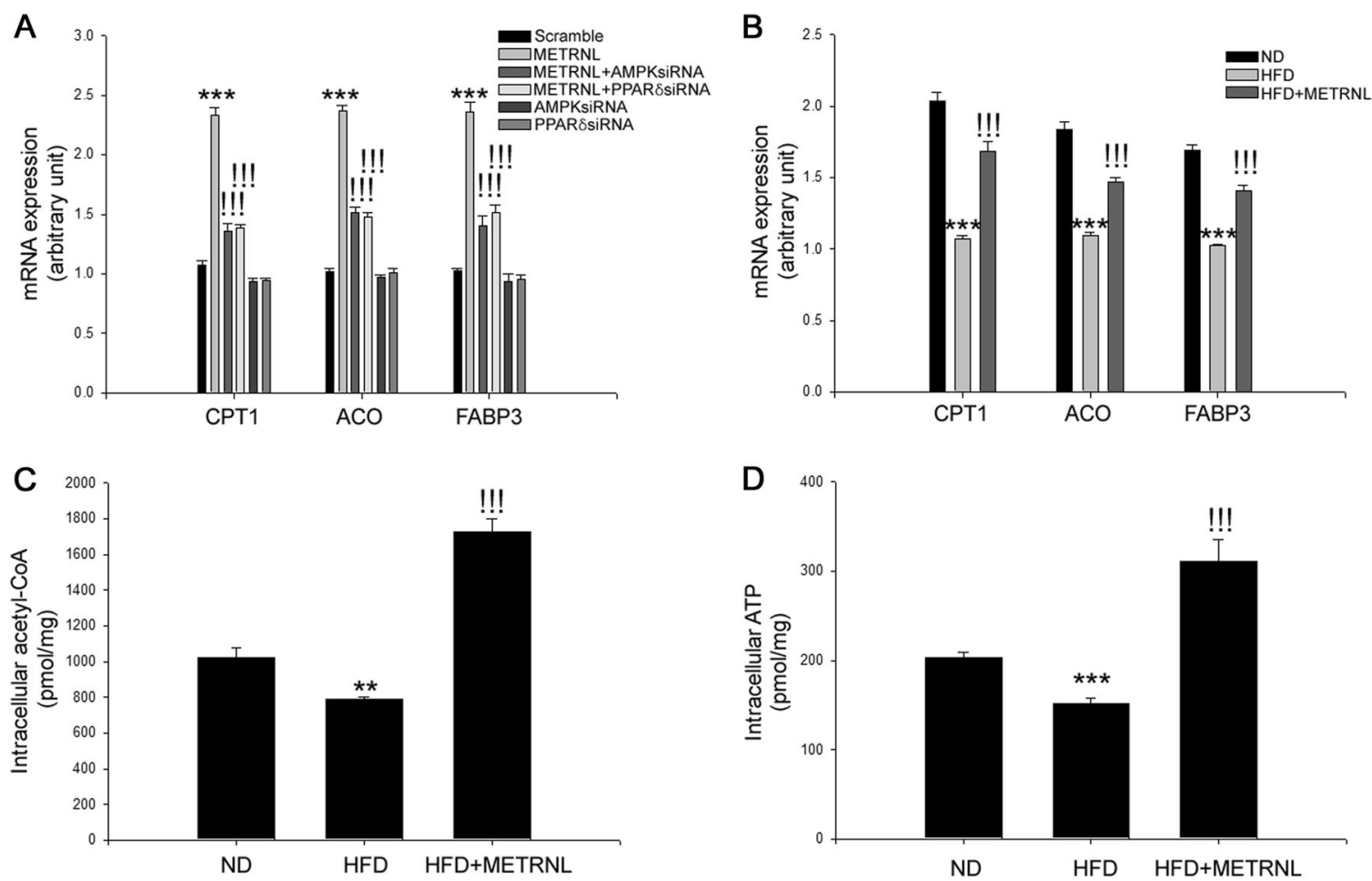

Fig. 6 METRNL stimulates fatty acid oxidation-associated gene expression. a Quantitative real-time PCR analysis of CPT1, ACO, and FABP3 mRNA expression in AMPK (20 nM) or PPARS siRNA (20 nM)-transfected C2C12 cells treated with METRNL (200 ng/mL) for $24 \mathrm{~h}$. b Quantitative realtime PCR analysis of CPT1, ACO, and FABP3 mRNA expression in HFD-fed mice treated with METRNL. $\mathbf{c}$ Intracellular acetyl-CoA and $\mathbf{d}$ ATP levels in soleus skeletal muscle of HFD-fed mice treated with METRNL $(1 \mu \mathrm{g} /$ mouse/day) for 8 weeks ( 5 animals/treatment group). Means \pm SEM were obtained from three separate experiments or five animals. ${ }^{* * *} P<0.001$ and ${ }^{* *} P<0.01$ compared to control or ND treatment. "!! $P<0.001$ compared to METRNL or HFD treatment

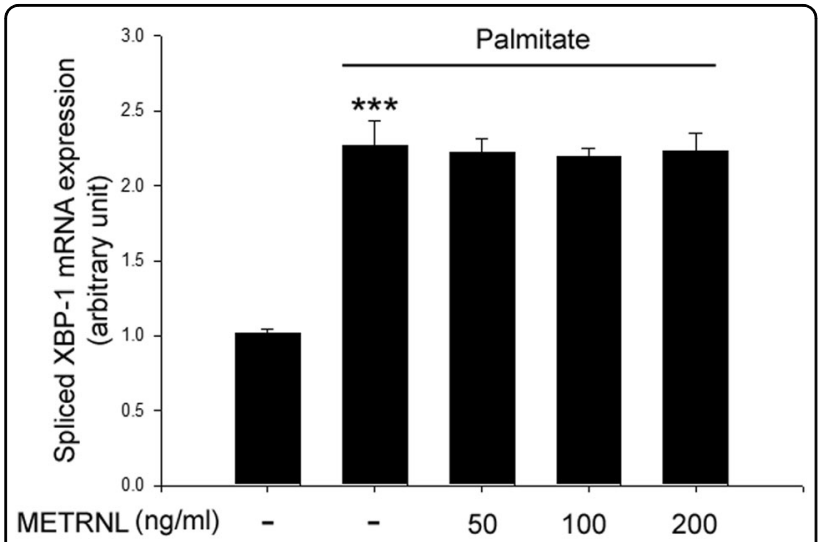

Fig. 7 METRNL did not affect palmitate-induced ER stress. Quantitative real-time PCR analysis of spliced XBP-1 mRNA expression in C2C12 cells treated with METRNL $(0-200 \mathrm{ng} / \mathrm{ml})$ for $24 \mathrm{~h}$. Means \pm SEM were obtained from three separate experiments. ${ }^{* * *} P<0.001$ compared to control

insulin resistance in myotubes. These results suggest and provide insight into one possible mechanism by which the positive effects of physical activity may be associated with METRNL-mediated AMPK signaling.
PPAR $\delta$ is known to be a critical regulator of the benefits of exercise in various organs, such as the heart, liver, fat, and skeletal muscle. Activation of PPAR $\delta$ ligand-mediated signaling ameliorates macrophageassociated inflammation and reduces serum triglyceride levels through modulation of lipoprotein metabolism and elevated plasma HDL-cholesterol levels. Activation of the PPAR $\delta$ pathway also mitigates hepatic gluconeogenesis, resulting in amelioration of hyperglycemia. Furthermore, PPAR $\delta$ pathway activation attenuates lipopolysaccharide-induced inflammation via suppression of NFKB-mediated signal transduction in cardiomyocytes $^{24}$. Fatty acid oxidation and skeletal muscle energy expenditure by PPAR $\delta$ bring many positive effects, such as weight loss, stimulation of skeletal muscle metabolic rate, attenuation of insulin resistance and atherogenic inflammation ${ }^{43}$. Thus, PPAR $\delta$ has emerged as a novel therapeutic target for metabolic disorders ${ }^{43}$. In the present study, we demonstrate for the first time that METRNL treatment can augment PPAR $\delta$ expression and consequently attenuate the inflammatory response via suppression of $\mathrm{NF \kappa B-mediated} \mathrm{signaling} \mathrm{and} \mathrm{pro-}$ inflammatory cytokines. 
Herein, METRNL-induced AMPK phosphorylation and PPAR $\delta$ expression decreased palmitate-induced inflammation. Saturated fatty acids have been previously suggested to cause inflammation through induction of proinflammatory cytokines, such as TNF $\alpha$ and IL-6, through NFkB activation ${ }^{44}$. In line with this finding, we reaffirmed that palmitate stimulates $\mathrm{NF \kappa B}$ activity, resulting in impairment of insulin signaling, such as IRS-1 and Akt phosphorylation, in differentiated $\mathrm{C} 2 \mathrm{C} 12$ cells. AMPK siRNA or PPAR $\delta$ markedly mitigated the inhibitory effects on palmitate-induced inflammation and insulin resistance, indicating that METRNL-induced AMPK and PPAR $\delta$ could contribute to the amelioration of inflammation and insulin resistance. In in vivo experiments using HFD-fed mice, METRNL treatment attenuated NFкB-mediated signaling and the levels of pro-inflammatory cytokines (TNF $\alpha$ and MCP-1). Additionally, we found that AMPK and PPAR $\delta$ are independently regulated by METRNL. These results suggest that METRNL alleviates palmitateor HFD-induced insulin resistance by ameliorating inflammation via AMPK- or PPAR $\delta$-dependent pathways in skeletal muscle. Furthermore, we demonstrated that METRNL increased PGC1 $\alpha$ expression through both AMPK- and PPAR $\delta$-mediated signaling. These results suggest the possibility that PGC1 $\alpha$ may play a central role in the METRNL-mediated attenuation of inflammation and insulin resistance in skeletal muscle. However, further studies are needed to elucidate this assumption.

The present study showed that METRNL administration improved both glucose tolerance and insulin tolerance in experimental mice. Elevated basal glucose levels were observed in HFD-fed mice during IPGTTs and ITTs. Therefore, the percentage of basal glucose levels was graphed to determine insulin sensitivity. Moreover, we demonstrated that METRNL administration suppressed basal glucose levels. These results may be associated with hepatic gluconeogenesis or glucose uptake by various organs through $\mathrm{AMPK}^{45,46}$, PPAR $\delta^{47,48}$, or other pathways.

In this study, METRNL treatment reduced the body weight of mice but did not influence calorie intake. These results suggest that body weight loss may be caused by fatty acid oxidation-mediated fat burning in adipose tissue and browning of white adipose tissue through the PGC1 $\alpha$ mediated pathway ${ }^{6}$, as METRNL-mediated internal mechanisms. However, no changes have been observed in body weight or energy expenditure in either METRNL transgenic mice or knockout mice ${ }^{7}$. This lack of an effect may be due to differences in experimental animal models and related conditions. Therefore, further studies are needed to investigate the effects of METRNL on fatty acid metabolism in adipose tissue. Additionally, METRNL did not attenuate palmitate-induced ER stress, which has been reported to play a causal role in the development of insulin resistance in skeletal muscle ${ }^{32}$, disclosing that the

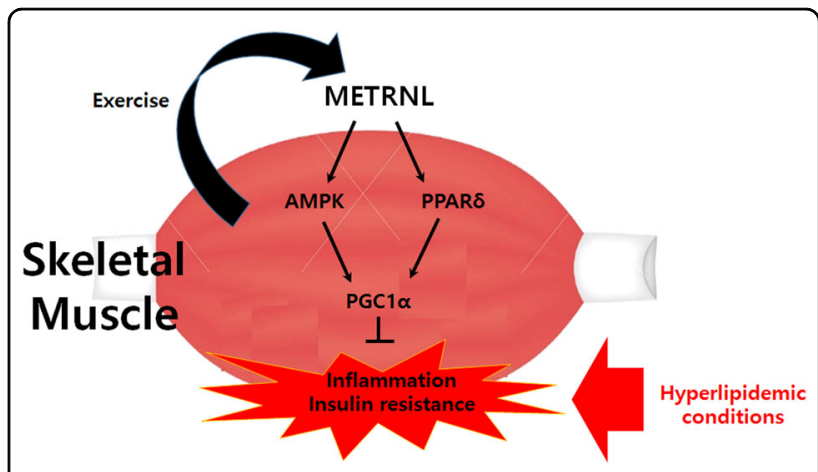

Fig. 8 Schematic diagram showing the effects of METRNL on skeletal muscle insulin resistance

protective effects of METRNL on inflammation and insulin resistance are not related to ER stress.

In conclusion, the current study is the first to show that METRNL ameliorates lipid-induced inflammation and insulin resistance via AMPK- or PPAR $\delta$-dependent signaling in skeletal muscle of mice (Fig. 8). These results may shed light on the novel mechanisms associated with the positive effects of physical activity and suggest an attractive therapeutic strategy for treating metabolic syndrome, including insulin resistance.

\section{Acknowledgements}

This study was supported by the Basic Science Research Program through the National Research Foundation of Korea (NRF) funded by the Ministry of Science, ICT \& Future Planning [2016R1C1B2012674].

\section{Author details}

${ }^{1}$ Research Administration Team, Seoul National University Bundang Hospital, Seongnam, Korea. ${ }^{2}$ College of Pharmacy, Chung-Ang University, Seoul, Korea. ${ }^{3}$ Neuropsychopharmacology and Toxicology Program, College of Pharmacy, Kangwon National University, Chunchon, Korea. ${ }^{4}$ College of Pharmacy, Sookmyung Women's University, Seoul, Korea. ${ }^{5}$ Department of Pharmacology, Faculty of Veterinary Medicine, Cairo University, 12211 Giza, Egypt.

${ }^{6}$ Department of Medical Pharmacology, Medical Faculty, Ataturk University, Erzurum, Turkey. 'Department of Pharmacology, College of Medicine, ChungAng University, 221, Heuksuk-dong, Dongjak-gu, Seoul 156-756, Korea

\section{Author contributions}

Design: T.W.J., S.H.L., H.-C.K., Y.K.S., A.M.A.E.-A., and J.H.J. Conducted/data collection: T.W.J., H.-C.K., and Y.K.S., S.H.L. Analysis: T.W.J., S.H.L., J.S.B. Writing of the manuscript: T.W.J., A.M.E.-A., and J.H.J.

Conflict of interest

The authors declare that they have no conflict of interest.

\section{Publisher's note}

Springer Nature remains neutral with regard to jurisdictional claims in published maps and institutional affiliations.

Supplementary information accompanies this paper at https://doi.org/ 10.1038/s12276-018-0147-5.

Received: 13 April 2018 Revised: 3 July 2018 Accepted: 25 July 2018. Published online: 13 September 2018 


\section{References}

1. Bogardus, C. et al. Effects of physical training and diet therapy on carbohydrate metabolism in patients with glucose intolerance and non-insulindependent diabetes mellitus. Diabetes 33, 311-318 (1984).

2. Schnyder, S. \& Handschin, C. Skeletal muscle as an endocrine organ: PGC1alpha, myokines and exercise. Bone 80, 115-125 (2015).

3. Li, Z. Y. et al. Subfatin is a novel adipokine and unlike Meteorin in adipose and brain expression. Cns. Neurosci. Ther. 20, 344-354 (2014).

4. Ushach, I. et al. METEORIN-LIKE is a cytokine associated with barrier tissues and alternatively activated macrophages. Clin. Immunol. 156, 119-127 (2015)

5. Zheng, S. L., Li, Z. Y., Song, J., Liu, J. M. \& Miao, C. Y. Metrnl: a secreted protein with new emerging functions. Acta Pharmacol. Sin. 37, 571-579 (2016).

6. Rao, R. R. et al. Meteorin-like is a hormone that regulates immune-adipose interactions to increase beige fat thermogenesis. Cell 157, 1279-1291 (2014).

7. Li, Z. Y. et al. Adipocyte metrnl antagonizes insulin resistance through PPARgamma signaling. Diabetes 64, 4011-4022 (2015).

8. Boden, G. Role of fatty acids in the pathogenesis of insulin resistance and NIDDM. Diabetes 46, 3-10 (1997).

9. Vessby, B. et al. Substituting dietary saturated for monounsaturated fat impairs insulin sensitivity in healthy men and women: the KANWU Study. Diabetologia 44, 312-319 (2001).

10. Coll, T. et al. Oleate reverses palmitate-induced insulin resistance and inflammation in skeletal muscle cells. J. Biol. Chem. 283, 11107-11116 (2008).

11. Senn, J. J. Toll-like receptor-2 is essential for the development of palmitateinduced insulin resistance in myotubes. J. Biol. Chem. 281, 26865-26875 (2006).

12. Shi, $H$. et al. TLR4 links innate immunity and fatty acid-induced insulin resistance. J. Clin. Invest. 116, 3015-3025 (2006).

13. Kim, J. K. et al. Prevention of fat-induced insulin resistance by salicylate. J. Clin. Invest. 108, 437-446 (2001).

14. Kern, P. A., Ranganathan, S., Li, C., Wood, L. \& Ranganathan, G. Adipose tissue tumor necrosis factor and interleukin-6 expression in human obesity and insulin resistance. Am. J. Physiol. Endocrinol. Metab. 280, E745-E751 (2001).

15. Bae, J. Y. Aerobic exercise increases meteorin-like protein in muscle and adipose tissue of chronic high-fat diet-induced obese mice. Biomed. Res. Int. 2018, 6283932 (2018).

16. Salminen, A., Hyttinen, J. M. \& Kaarniranta, K. AMP-activated protein kinase inhibits NF-kappaB signaling and inflammation: impact on healthspan and lifespan. J. Mol. Med (Berl.) 89, 667-676 (2011).

17. Zhang B, B., Zhou, G. \& Li, C. AMPK: an emerging drug target for diabetes and the metabolic syndrome. Cell. Metab. 9, 407-416 (2009).

18. Qin, Z. Y. et al. Metformin prevents LYRM1-induced insulin resistance in 3T3-L1 adipocytes via a mitochondrial-dependent mechanism. Exp. Biol. Med. 239, 1567-1574 (2014).

19. Jager, S., Handschin, C., St-Pierre, J. \& Spiegelman, B. M. AMP-activated protein kinase (AMPK) action in skeletal muscle via direct phosphorylation of PGC1alpha. Proc. Natl Acad. Sci. USA 104, 12017-12022 (2007).

20. Handschin, C. \& Spiegelman, B. M. The role of exercise and PGC1alpha in inflammation and chronic disease. Nature 454, 463-469 (2008).

21. Kleiner, S. et al. Development of insulin resistance in mice lacking PGC-1alpha in adipose tissues. Proc. Natl Acad. Sci. USA 109, 9635-9640 (2012).

22. Lee, W. J. et al. AMPK activation increases fatty acid oxidation in skeletal muscle by activating PPARalpha and PGC-1. Biochem. Biophys. Res. Commun 340, 291-295 (2006)

23. Narkar, V. A. et al. AMPK and PPARdelta agonists are exercise mimetics. Cell 134, 405-415 (2008).

24. Ding, G., Cheng, L., Qin, Q., Frontin, S. \& Yang, Q. PPARdelta modulates lipopolysaccharide-induced TNFalpha inflammation signaling in cultured cardiomyocytes. J. Mol. Cell. Cardiol. 40, 821-828 (2006).
25. Kilgore, K. S. \& Billin, A. N. PPARbeta/delta ligands as modulators of the inflammatory response. Curr. Opin. Investig. Drugs 9, 463-469 (2008).

26. Clark, R. B. The role of PPARs in inflammation and immunity. J. Leukoc. Biol. 71 388-400 (2002).

27. Lee, C. H. et al. PPARdelta regulates glucose metabolism and insulin sensitivity. Proc. Natl Acad. Sci. USA 103, 3444-3449 (2006).

28. Barish, G. D., Narkar, V. A. \& Evans, R. M. PPAR delta: a dagger in the heart of the metabolic syndrome. J. Clin. Invest. 116, 590-597 (2006).

29. Hondares, E.et al. PPARdelta, but not PPARalpha, activates PGC-1alpha gene transcription in muscle. Biochem. Biophys. Res. Commun. 354, 1021-1027 (2007)

30. Koves, T. R. et al. Mitochondrial overload and incomplete fatty acid oxidation contribute to skeletal muscle insulin resistance. Cell. Metab. 7, 45-56 (2008).

31. Tanaka, T. et al. Activation of peroxisome proliferator-activated receptor delta induces fatty acid beta-oxidation in skeletal muscle and attenuates metabolic syndrome. Proc. Natl Acad. Sci. USA 100, 15924-15929 (2003).

32. Peng, G. et al. Oleate blocks palmitate-induced abnormal lipid distribution, endoplasmic reticulum expansion and stress, and insulin resistance in skeletal muscle. Endocrinology 152, 2206-2218 (2011).

33. Ruas, J. L. et al. A PGC-1alpha isoform induced by resistance training regulates skeletal muscle hypertrophy. Cell 151, 1319-1331 (2012).

34. Salt, I. P. \& Palmer, T. M. Exploiting the anti-inflammatory effects of AMPactivated protein kinase activation. Expert. Opin. Investig. Drugs 21, 1155-1167 (2012).

35. Ruderman, N. B., Carling, D., Prentki, M. \& Cacicedo, J. M. AMPK, insulin resistance, and the metabolic syndrome. J. Clin. Invest. 123, 2764-2772 (2013).

36. Wu, Y., Song, P., Xu, J., Zhang, M. \& Zou, M. H. Activation of protein phosphatase 2A by palmitate inhibits AMP-activated protein kinase. J. Biol. Chem. 282, 9777-9788 (2007).

37. Boden, $\mathrm{G}$. Effects of free fatty acids (FFA) on glucose metabolism: significance for insulin resistance and type 2 diabetes. Exp. Clin. Endocrinol. Diabetes 111, 121-124 (2003).

38. Boden, G. Free fatty acids, insulin resistance, and type 2 diabetes mellitus. Proc. Assoc. Am. Physicians 111, 241-248 (1999).

39. de Carvalho Vidigal, F., Guedes Cocate, P., Goncalves Pereira, L., de Cassia \& Goncalves Alfenas, R. The role of hyperglycemia in the induction of oxidative stress and inflammatory process. Nutr. Hosp. 27, 1391-1398 (2012).

40. Cardaci, S., Filomeni, G. \& Ciriolo, M. R. Redox implications of AMPKmediated signal transduction beyond energetic clues. J. Cell. Sci. 125, 2115-2125 (2012)

41. Salvado, L. et al. PPARbeta/delta prevents endoplasmic reticulum stress-associated inflammation and insulin resistance in skeletal muscle cells through an AMPK-dependent mechanism. Diabetologia 57, 2126-2135 (2014).

42. Musi, N. et al. AMP-activated protein kinase (AMPK) is activated in muscle of subjects with type 2 diabetes during exercise. Diabetes 50, 921-927 (2001).

43. Reilly, S. M. \& Lee, C. H. PPAR delta as a therapeutic target in metabolic disease. FEBS Lett. 582, 26-31 (2008)

44. Tripathy, D. et al. Elevation of free fatty acids induces inflammation and impairs vascular reactivity in healthy subjects. Diabetes 52, 2882-2887 (2003).

45. Mihaylova, M. M. \& Shaw, R. J. The AMPK signalling pathway coordinates cell growth, autophagy and metabolism. Nat. Cell Biol. 13, 1016-1023 (2011).

46. Musi, N. \& Goodyear, L. J. AMP-activated protein kinase and muscle glucose uptake. Acta Physiol. Scand. 178, 337-345 (2003).

47. Souza-Mello, V. Peroxisome proliferator-activated receptors as targets to treat non-alcoholic fatty liver disease. World J. Hepatol. 7, 1012-1019 (2015).

48. Kramer, D. K. et al. Direct activation of glucose transport in primary human myotubes after activation of peroxisome proliferator-activated receptor delta. Diabetes 54, 1157-1163 (2005). 